Preprint

UCRL-JC-138060

\title{
Confocal Imaging of Biological Tissues Using Second Harmonic Generation
}

B.-M. Kim, P. Stoller, K. Reiser, J. Eichler, M. Yan, A. Rubenchik, L. Da Silva

This article was submitted to

U.S. Department of Energy

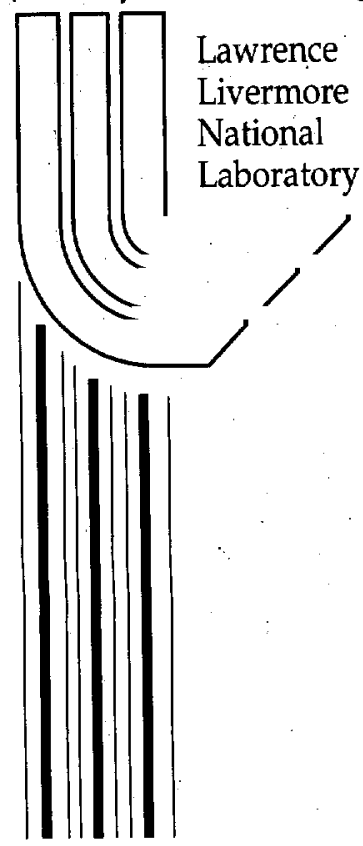

Photonics West, San Jose, CA, January 23-29, 2000

\section{March 6, 2000}




\section{DISCLAIMER}

This document was prepared as an account of work sponsored by an agency of the United States Government. Neither the United States Government nor the University of California nor any of their employees, makes any warranty, express or implied, or assumes any legal liability or responsibility for the accuracy, completeness, or usefulness of any information, apparatus, product, or process disclosed, or represents that its use would not infringe privately owned rights. Reference herein to any specific commercial product, process, or service by trade name, trademark, manufacturer, or otherwise, does not necessarily constitute or imply its endorsement, recommendation, or favoring by the United States Government or the University of California. The views and opinions of authors expressed herein do not necessarily state or reflect those of the United States Government or the University of California, and shall not be used for advertising or product endorsement purposes.

This is a preprint of a paper intended for publication in a journal or proceedings. Since changes may be made before publication, this preprint is made available with the understanding that it will not be cited or reproduced without the permission of the author.

This report has been reproduced directly from the best available copy.

Available to DOE and DOE contractors from the Office of Scientific and Technical Information

P.O. Box 62, Oak Ridge, TN 37831

Prices available from (423) 576-8401 http://apollo.osti.gov/bridge/

Available to the public from the National Technical Information Service

U.S. Department of Commerce 5285 Port Royal Rd., Springfield, VA 22161 http://www.ntis.gov/

OR

Lawrence Livermore National Laboratory Technical Information Department's Digital Library http://www.llnl.gov/tid/Library.html 


\title{
Confocal Imaging of Biological Tissues Using Second Harmonic Generation
}

\author{
Beop-Min Kim ${ }^{1}$, Patrick Stoller ${ }^{1}$, Karen Reiser ${ }^{2}$, Jürgen Eichler ${ }^{3}$, Ming Yan', \\ Alexander Rubenchik', Luiz Da Silva' \\ ${ }^{1}$ Medical Technology Program, Lawrence Livermore National Laboratory, Livermore, CA \\ ${ }^{2}$ University of California Medical Center at Davis, Davis, CA \\ ${ }^{3}$ Technische Fachhochschule-Berlin, Berlin Germany
}

\begin{abstract}
A confocal microscopy imaging system was devised to selectively detect second harmonic signals generated by biological tissues. Several types of biological tissues were examined using this imaging system, including human teeth, bovine blood vessels, and chicken skin. All these tissues generated strong second harmonic signals. There is considerable evidence that the source of these signals in tissue is collagen. Collagen, the predominant component of most tissues, is known to have second order nonlinear susceptibility. This technique may have diagnostic usefulness in pathophysiological conditions characterized by changes in collagen structure including malignant transformation of nevi, progression of diabetic complications, and abnormalities in wound healing.
\end{abstract}

\section{Introduction}

The predominant structural protein in animals is collagen, a macromolecule comprising at least 17 genetically distinct forms. Collagen types are generally classified as either fibrillar or nonfibrillar. Fibrillar collagens, such as types I, II, and III, comprise the major structural collagens of the body. They are characterized at the molecular level by a triple helical structure, and at the supramolecular level by ordered arrays of fibrils and bundles [1]. They lack inversion symmetry which is a necessary condition for second order nonlinear susceptibility. The second order nonlinearity of the collagen is responsible for generation of light at twice the excitation frequency (second harmonic generation) when interacting with intense laser pulses [2]-[5]. The development of ultrashort pulse laser technology has made it feasible to analyze harmonic generation in collagen nondestructively. Recently, it was reported that femtosecond laser pulses generate second harmonic light in collagen more effectively without damaging the target tissues than picosecond or nanosecond pulses [5]. Since collagen is the only significant source of second harmonic signals in tissues, nonlinear optical imaging offers the possibility of obtaining structural information about collagen with exceptionally high signal-to-noise ratios. In the present study, we report the 
development of a nonlinear optical imaging system capable of nondestructive analysis of biological samples, in which we combine the resolution of confocal microscopy with the specificity of second harmonic signal detection. We further report that this system can be successfully used on a diverse range of soft and hard biological tissues, including tooth, skin, and blood vessel.

\section{Materials and Methods:}

We used a Ti:Sapphire (Spectra-Physics Tsunami) ocscillator pumped by a $5 \mathrm{~W}$, frequency-doubled Nd-YAG (Spectra-Physics Merlin) laser to produce $100 \mathrm{fs}, 800 \mathrm{~nm}$ pulses at a repetition rate of $82 \mathrm{MHz}$. The laser beam passes through a $1.5 \mathrm{kHz}$ optical chopper for lock-in amplification of the signal. The laser intensity is controlled using a half-wave plate and a birefringent calcite crystal. The laser beam is focused onto a sample mounted on a translation stage using a $20 \mathrm{~mm}$ focal length microscope objective (Mitutoyo) with a numerical aperture of 0.42 . This produces a focal spot with approximately 1 micron diameter and an approximate beam waist of 10 microns.

The back-scattered light is collected by the same microscope objective and transmitted through the dichroic mirror that reflects the input beam onto the sample. The signal is focused through a 50 micron pinhole using an $\mathrm{f}=50 \mathrm{~mm}$ lens, collimated by another lens, and then reflected onto a photomultiplier tube (PMT) using a series of dichroic mirrors that reject two-photon fluorescence and first-harmonic light. The use of a confocal detection system allows improved depth resolution by eliminating SHG generated away from the focal spot.

Some of the back-scattered first-harmonic light is reflected at one of the calcite crystal interfaces and detected using a photodiode. The PMT and photodiode signals are lock-in amplified using the reference signal from the optical chopper. A Labview program is used to move the motorized translation stage and to collect data from the lock-in amplifiers.

Chicken skin (kept frozen at $-80^{\circ}$ ) was obtained from previous studies. Human tooth samples, containing carious lesions, were obtained from UCSF School of Dentistry. Bovine artery was dissected from commercially obtained bovine shanks. For analysis, all samples were clamped between two glass slides and mounted on the translation stage. Samples were kept moist with physiological saline. Several scans were performed on each sample in order to determine reproducibility of results.

\section{Experimental Results}

Hard Tissue (dentin): We made several scans of the surface of a tooth at the boundary between healthy dentin and cavity. Fig. 1(a) below shows the strong contrast 
between dentin and cavity in the second harmonic signal. Fig. 1(b) gives the first harmonic signal obtained from the same scan. While the contrast ratio in the SHG scan is 180:1 between dentin and cavity, it is only 5:1 in the first harmonic scan.

Bovine Arteries: Arteries were analyzed in several ways. We cut out a thin (about 2 $\mathrm{mm}$ ) cross-section of the artery (Fig. 2) and performed several one-dimensional scans across the surface, moving from the hole in the center to the outer edge. Fig. 3 is an illustration of the cross-section of an artery [1]. As shown in Fig. 4, the SHG signal is weaker near the endothelium, the elastic lamina and the tunica media, which is a layer composed mostly of soft muscle tissues. The SHG intensity rapidly increases as the beam focus approaches the adventitia, which is rich in collagen content. The signal from the adventita is by no means uniform; the large fluctuations in the SHG signal suggest that our detection technique is able to resolve features on the scale of a few microns. When we repeated the scan (Fig. 4(a) and 4(b)), we found that results were highly replicable, suggesting that fluctuations in signal intensity represent determinant features and not random noise. The repeated scans also confirmed that the high intensity laser pulses do not appear to damage the artery tissue.

Chicken Skin: We performed two-dimensional scans on chicken skin (Fig. 5). Fluctuation in SHG intensity gave the appearance of a layered structure with distinct structural features, especially near the left side of the scan. There are also features about 50 microns deep (chicken surface not shown in the figure) and about 10 to 20 microns across in the SHG scan. The features are separated by about 10 to 20 microns. We use the peak in the first harmonic signal that occurs when the focus of the beam crosses the glass slidechicken skin interface to indicate the location of the surface. This technique confirms that we are observing second harmonic generation from just inside the chicken skin and not from the surface.

\section{Discussion}

In the present study we have shown that it is possible to obtain second harmonic images from a variety of biological tissues using confocal microscopy linked to a femtosecond laser. Such images have very high-resolution (on the order of microns) and high replicability. We also found that there was no evidence of tissue destruction. We were able to perform two-dimensional scans, similar to those described by Guo et al. [6;7] in which imaging is simultaneously obtained along the surface and into the sample.

These studies suggest that detailed studies of collagen are feasible using second harmonic analysis. A major advantage of this technique is high specificity, since light is generated only from material that possesses second order nonlinear susceptibility. Thus, 
targeted imaging is possible even when this nonlinear material is embedded inside turbid media. As demonstrated by our preliminary studies and by other studies in the literature, collagen can be selectively imaged inside the tissue. The intensity of the second harmonic signal is highly dependent on the chirality of the collagen fibrils, a structural feature that is known to change in specific pathologic conditions. For example, conditions involving partial degradation of collagen, increased nonenzymatic glycation, and/or glyco-oxidative damage result in a decrease in chiral structure. We might therefore expect to see decreased SHG intensity in diabetes, aging, and during neoplastic transformation of nevi into melanoma. Conversely, conditions such as hypertrophic scarring and scleroderma, which are characterized by an increase in enzymatically mediated crosslinking, result in an increase in chirality, and we would therefore expect to see an increase in SHG intensity.

\section{ACKNOWLEDGMENTS}

This work was performed at Lawrence Livermore National Laboratory under the auspices of the U.S. department of Energy under contract No. W-7405-ENG-48.

\section{References}

1. D. W. Fawcett, A Textbook of Histology (Chapman and Hall, New York, 1994), p. 133.

2. I. Freund, M. Deutsch, and A. Sprecher, "Connective tissue polarity: optical secondharmonic microscopy, cross-beam summation, and small-angle scattering in rat-tail tendon," Biophys. J. 50,693-712 (1986).

3. G. B. Altshuler, N. R. Belashenkov, G. A. Martsinovski, and A. A. Solounin, "Nonlinear transmission and second-harmonic generation in dentin in the field of ultrashort Nd-laser pulses," in Advanced Laser Dentistry, G. B. Altshuler, R. J. Blankenau, and H. A. Wigdor, eds., Proc. SPIE 1984, 6-10 (1995).

4. V. Hovanessian and A. Lalayan, "Second-harmonic generation in biofiber-containing tissues," in Proceedings of the International conference on Lasers'96 (Society for Optical and Quantum Electronics, McLean, VA., 1996), pp. 107-110.

5. B.-M. Kim, J. Eichler, and L. B. Da Silva, "Frequency doubling of ultrashort pulses in biological tissues," Appl. Opt. 38, 7145-7150 (1999).

6. Y. Guo, P. P. Ho, H. Savage, D. Harris, P. Sacks, S. Schantz, F. Liu, N. Zhadin, and R. R. Alfano, "Second-harmonic tomography of tissues," Opt. Lett. 22, 1323-1325 (1997).

7. Y. Guo, P. P. Ho, A. Tirksliunas, F. Liu, and R. R. Alfano, "Optical harmonic generation from animal tissues by the use of picosecond and femtosecond laser pulses," Appl. Opt. 35, 6810-6813 (1996). 

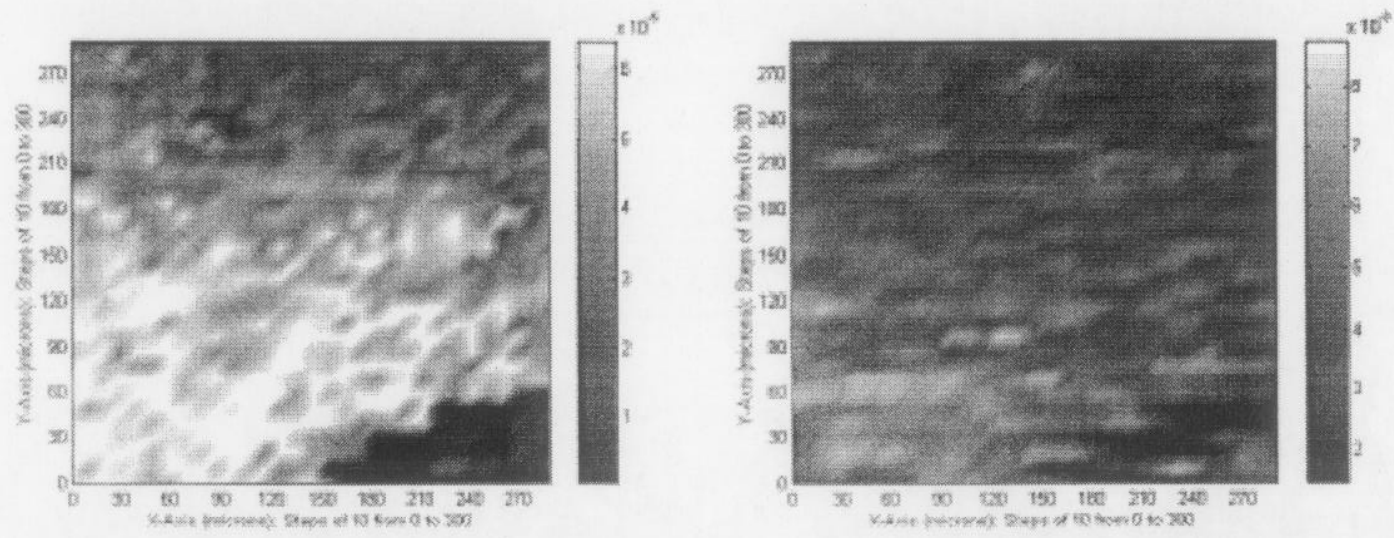

Fig. 1. (a) SHG scan of cavity/dentin boundary. (b). First harmonic scan of the cavity/ dentin boundary. The cavity is located in the lower-right corner of the scan.

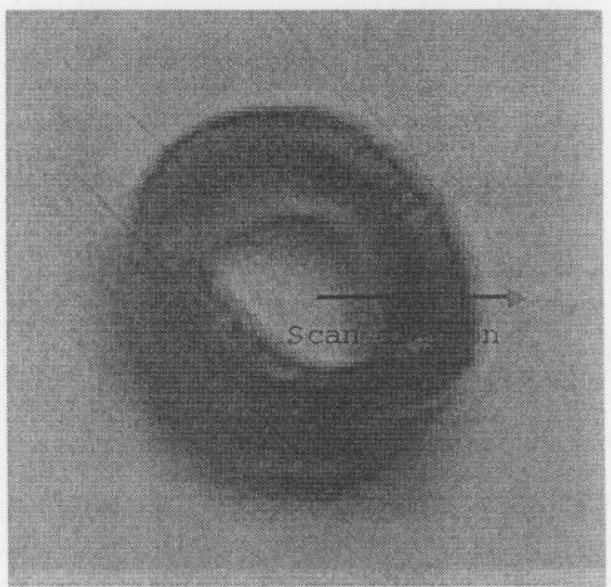

Fig. 2. Photograph of bovine artery crosssection showing scan direction.

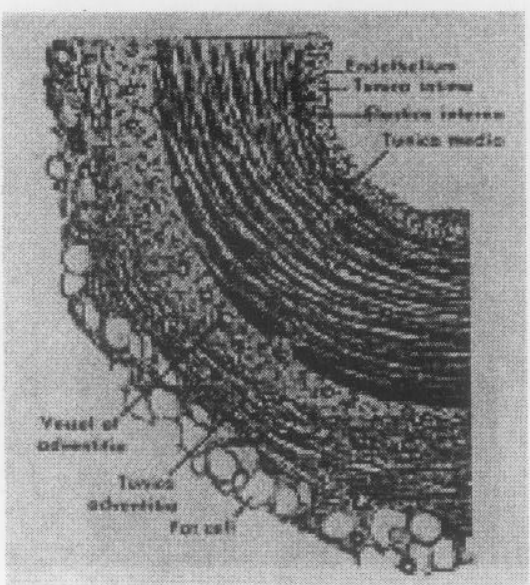

Fig. 3. Illustration of structure of artery [1]. 


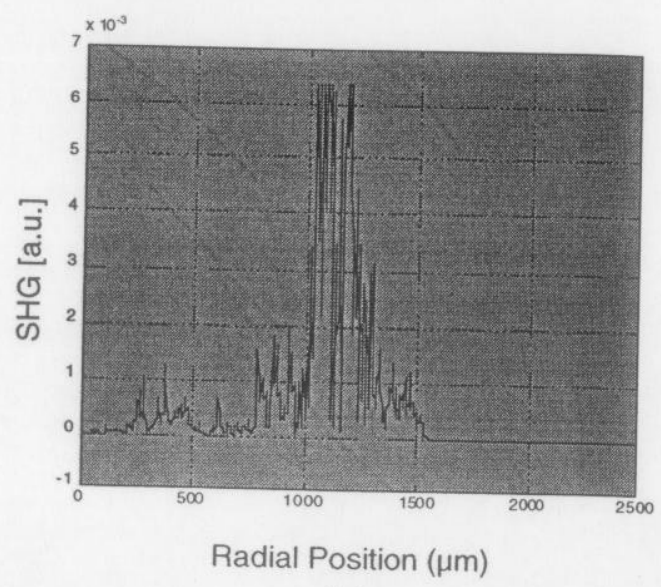

(a)

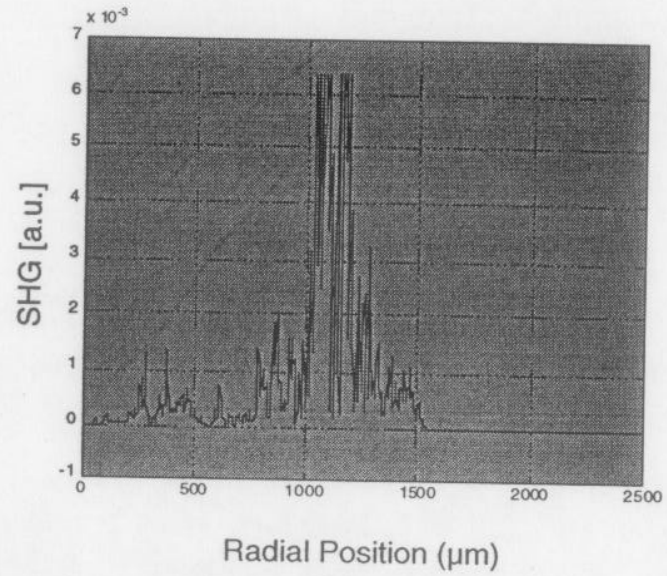

(b)

Figure 4. (a) Scans of cross-section of bovine artery. $x=0$ microns corresponds to the center area and $\mathrm{x}$ around 1500 microns corresponds to the outer edge. Note that the lock-in amplifier saturated; the highest peaks actually indicate a higher signal than recorded (b). Repeat of scan shown in Fig. 4(a). Note that the same features can be observed in both scans.

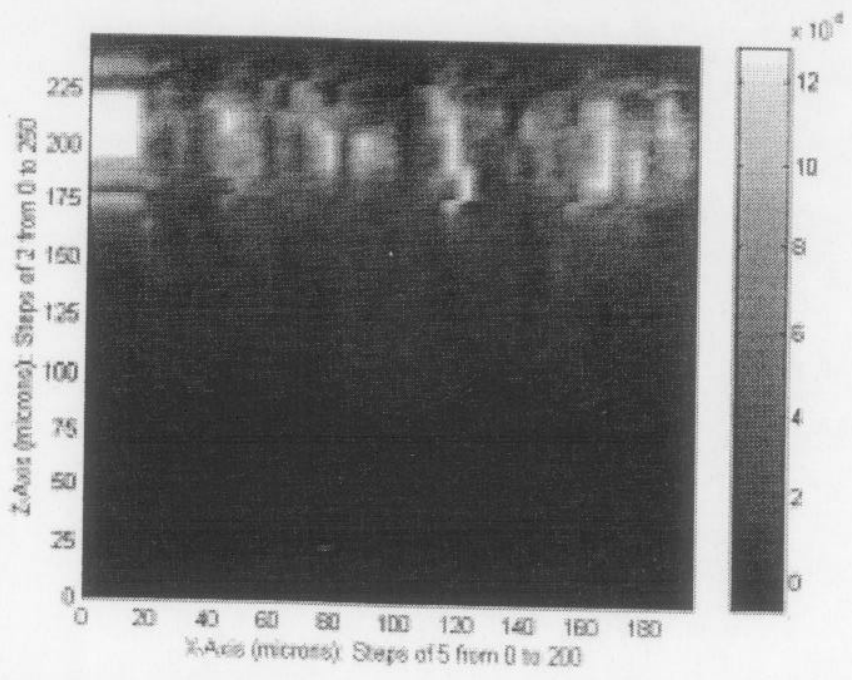

Figure 5. Confocal scan of chicken skin. $\mathrm{x}$-axis is along the surface and positive $\mathrm{z}$-axis points along the normal to the skin surface. The surface is at about 250 microns; lower values of $\mathrm{z}$ are inside the chicken skin. 